\title{
Effect of Eccentric Isotonic Quadriceps Muscle Exercises on Patellofemoral Pain Syndrome: An Exploratory Pilot Study
}

Charu Eapen*, PhD; Chetan D. Nayak, MPT; Chundanveetil Pazhyaottyil Zulfeequer, MPT

Authors' Affiliation:

Department of Physiotherapy

KMC Mangalore

Manipal University, India

* Corresponding Author;

Address: Department Physiotherapy, KMC Mangalore, 575003

Manipal University, India

E-mail: charu_mak@hotmail.com

Received: Apr 05, 2011

Accepted: Jun 17, 2011

Key Words: Quadriceps Muscle; Patellofemoral Pain Syndrome; Isotonic Contraction; Eccentric Exercises; Muscle Strength

\begin{abstract}
Purpose: There is a decrease in quadriceps muscle strength in subjects with patellofemoral pain syndrome. Various types of strengthening exercises of the quadriceps are done as part of its management, but the effect of isotonic eccentric quadriceps muscle exercises on patellofemoral pain syndrome has not been studied. Hence the aim of this exploratory pilot study was to evaluate the effect of eccentric quadriceps training in patients with patellofemoral pain.

Methods: Twenty patients (12 female and 8 male, mean ages, 27.50 +/- 6.6 years) with patellofemoral pain syndrome were treated. The eccentric training of the quadriceps was given using a Baltimore Therapeutic Equipment (BTE) Primus machine. The main outcome measures used were percentage time on target as shown by the BTE primus machine, SF-36 Health questionnaire and patellofemoral pain severity scale. Statistical analysis was performed using SPSS 13.
\end{abstract}

Results: All the outcome measures showed significant improvements $(P<0.05)$. Percentage time on target improved with a mean difference of 23.6, the SF-36 questionnaire showed an improvement in physical component score, mental component score and bodily pain with a mean difference of 10.9, 2.6 and 29.2 respectively and pain score when taken using patellofemoral severity scale also improved with a mean difference of 3.4.

Conclusion: Isotonic eccentric training of quadriceps muscles was found to be effective in reducing pain and improving the functional status of patients with patellofemoral pain syndrome and can be suggested as part of the treatment.

Asian Journal of Sports Medicine, Volume 2 (Number 4), December 2011, Pages: 227-234

\section{INTRODUCTION}

$\mathrm{P}$ atellofemoral disorders are probably the most common knee pathology encountered by the orthopaedic and sports medicine clinicians ${ }^{[1]}$. Anterior knee pain is one of the most common conditions seen in the outpatient physical therapy clinic ${ }^{[2]}$ and is one of the most difficult to treat. Reports of patellofemoral pain incidence in the clinical environment range from 21 to $40 \%{ }^{[3]}$. Patellofemoral pain syndrome (PFPS) is the most common diagnosis in outpatients presenting with anterior knee pain ${ }^{[4]}$. It affects as much as $25 \%$ of the general nonathletic population and occurs in all age groups and more common among adolescents and young adults ${ }^{[5]}$.

Patellofemoral pain is usually described as diffuse and poorly localized to the anterior aspect of the knee. Symptoms are typically aggravated by activities like descending stairs and relieved by rest. The mechanism for PFPS is not well understood; however, it has been 
suggested that the condition may arise from abnormal muscular and biomechanical factors that alter tracking of the patella within the femoral trochlear notch, contributing to increased patellofemoral contact pressures that result in pain and dysfunction ${ }^{[6]}$.

Muscle dysfunction (e.g. quadriceps weakness, improper firing pattern) is one of the major factors leading to patellofemoral pain ${ }^{[4]}$. This is altered motor control of the quadriceps muscle and reduction in the force producing capabilities of the Vastus medialis obliquus (VMO) ${ }^{[7,8]}$. Atrophy and weakness of the quadriceps muscle is also found universally with PFPS ${ }^{[9,10]}$. Isokinetic studies have shown that knee extension peak torque values were lower at PFPS when compared with those of healthy controls indicating decrease in the quadriceps muscle's strength in the subjects with PFPS ${ }^{[11-13]}$.

Much of PFPS rehabilitation focuses on quadriceps strengthening ${ }^{[14,15]}$. Quadriceps femoris muscle strengthening, is useful for improving functional ability, and may be particularly important for individuals who want to return to higher-demand activities such as running or other activities ${ }^{[16]}$. Previous studies have emphasized the importance of quadriceps strengthening in patients with patellofemoral pain ${ }^{[11,16-19]}$. A recent study has shown that a large number of PFPS patients can experience significant improvements in pain, function and quality of life, at least in the short term, with quadriceps femoris rehabilitation, with or without emphasis on selective activation of the VMO component ${ }^{[20]}$.

In one study it was seen that a majority of PFPS patients failed to smoothly control eccentric quadriceps contraction during stair descent or open kinetic chain isokinetic action. There appears to be a relationship between PFPS and control of eccentric quadriceps contraction ${ }^{[7,8]}$. In another study by Thomee R, a small increase in average torque were found during eccentric contractions across a limited range in the group using eccentric contractions compared with the group using isometric contractions ${ }^{[21]}$. Hence there is a need for eccentric training particularly among these patients with PFPS.

There are various methods of strengthening the quadriceps muscles like isometric exercises, isokinetic training and isotonic mode. Since the patients often complain of knee pain during dynamic phases such as stair climbing, squatting, walking and running, it seems more appropriate to strengthen their quadriceps muscle during dynamic exercises and dynamic muscle training primarily increases the dynamic function. Eccentric training can be given in the isotonic and isokinetic way $^{[11]}$. The eccentric isotonic exercises constitute a vital part in the muscular strengthening program as a weakness of the muscles in the eccentric phase could increment reactional forces in the patellofemoral joint. Also, it has been found that patients with anterior knee pain and patellofemoral instability develop a larger torsional movement in the quadriceps concentric contraction than in the eccentric one ${ }^{[22]}$. Isotonic exercise also has the advantage of being more functional than isokinetic exercise.

To our knowledge effect of isotonic eccentric quadriceps exercises on patellofemoral pain syndrome has not been studied though a relationship has been seen between the PFPS and eccentric quadriceps contraction. Hence, there was a need to study this aspect to help in clinical decision making.

The aim of this exploratory pilot study was thus to evaluate the effect of eccentric quadriceps training in patients with patellofemoral pain who complain that their pain is particularly associated with decelerating movements of the quadriceps muscle.

\section{METHODS AND SUBJECTS}

This was an exploratory pilot study to determine if a subsequent randomized controlled trial would be appropriate. The study was approved by the scientific committee of the institution and was conducted from August 2007 to August 2008. A total of 24 subjects were included, who were taken from the inpatient and the outpatient departments of KMC Hospital, Mangalore, India. 4 patients were lost in the follow up and were excluded from the study. Twenty patients completed the study from which 8 subjects were male and 12 subjects were female. The mean age was 27.50 \pm 6.6 . Fifty percent of the patients were in the age group of 21-25years, 35\% between 26-30 years and the 
remaining above 31 years. Patients who were diagnosed by an orthopedic surgeon to have PFPS, unilaterally or bilaterally and who had not under gone any strengthening program of lower limb for the last 6 weeks were included for the study. The inclusion criteria were: (a) anterior knee pain present during at least two of the following: ascending/descending stairs, hopping/running, squatting, kneeling and prolonged sitting; (b) insidious onset of symptoms unrelated to a traumatic incident; (c) presence of pain on step down from a 25-cm step or double-leg squat; and (d) pain on palpation of patellar facets. Subjects were excluded if they had: (a) symptoms present for <1 month; (b) clinical evidence of other knee pathology (including coexisting pathology); (c) undergone a previous knee surgery; (d) history of patellar fracture/subluxation/ dislocation; (e) current significant injury affecting other lower limb joints; (f) current use of nonsteroidal anti-inflammatory drugs or corticosteroids; (g) malignancy; (h) presence of severe pain or discomfort during testing, which prevents further participation of the individual in the study.

The outcome measures were Percentage time on target, the PFPS Severity Scale (PSS) and Short form health survey-36 (SF 36). All the patients were trained on Isokinetic Dynamometer - Baltimore Therapeutic Equipment (BTE) Primus RS.

\section{Test procedures}

Informed consent was obtained from all the patients. Demographic data was collected; baseline measurement of pain was recorded using the PSS. This scale is found to be reliable and has demonstrated convergent validity making it a useful tool for monitoring rehabilitative or surgical outcomes in clients with PFPS ${ }^{[23]}$. It has two subscales; one representing average pain, and one encompassing the subject's ability to perform functional activities. The 1st subscale was a five-point Likert scale on which the subject was asked to rate the pain they would experience in a typical week ( $0=$ none, $1=$ mild, $2=$ moderate, 3 = severe, $4=$ extreme). The second subscale was made up of eight items in visual analogue scale format which asked the subject to indicate how well they were able to perform the following activities over the past week. These included: climbing stairs, squat-ting down, walking, jogging, running/sprinting, participating in a sport, sitting with knees bent (for 20 minutes), and kneeling on knees for any time period. The maximum score for each statement is ' 10 ' (or 10 $\mathrm{cm}$ ), giving a total maximum score of ' 100 'for the PSS Health status of the patient was assessed by asking patients to fill SF-36 health survey questionnaire. Isokinetic dynamometry was performed to evaluate eccentric quadriceps action. Before the session of exercise started, all visits began with three minutes' warm up on a static exercise bicycle followed by 30 seconds of stretching of the quadriceps and hamstring muscles. A standard familiarization session was done on biceps brachii muscle, which included a detailed explanation of the differences between concentric and eccentric contractions and the subjects were given the opportunity to experience how the muscle would be acting during the training. The same investigator performed all the tests.

The patient was made to sit on the treatment chair. Standard stabilization strapping was placed across the distal thigh and the chest. The length of dynamometer was made suitable with the length of knee of each patient. Continuous passive motion (CPM) mode was used for the treatment. The session was started by beginning the CPM and cycled through 2 repetitions, to determine the weight of the limb by viewing the scale on the left side of the graph. Then the target force corresponding to $20 \%$ more than the highest peak of the graph (weight of the limb) was set. The patient was then asked to attempt to control the limb through the set ROM to achieve a “\% time on target” (Percentage time on target) that is $50 \%$ or greater for the 10 minute duration of exercise at $10 \mathrm{deg} / \mathrm{sec}$. Percentage time on target is the percentage of the total treatment time that a patient will be able to control the target force effectively.

The computer monitor was positioned so that the patient could see it and exert force so that graphing line stays between the horizontal dotted lines on the screen. When the target is set, the color of the feedback line on the screen indicates the direction in which the patient is applying the force. The red color feedback line on the screen indicated that the patient is applying force against the moving attachment as if trying to stop it (eccentric contraction of the quadriceps). According to 
Table 1: Changes in the "percentage time on target" values

\begin{tabular}{|cccc|} 
& $\begin{array}{c}\text { Mean } \\
\text { (Standard Deviation) }\end{array}$ & $\begin{array}{c}\text { Mean } \\
\text { Difference }\end{array}$ & P value \\
\hline Pre & $27.10(13.75)$ & -23.65 & $<0.001$ \\
Post & $50.75(15.84)$ & & \\
\hline
\end{tabular}

the SPEED protocol (Successive Progression of Exercise using Empiric Decision-making) of the BTE technologies it should take approximately 3-6 visits to achieve this. Hence a total of six visits were planned. After the patient finished the session of exercise during each visit the percentage time on target was seen as displayed on the screen. All the patients underwent the same sequence of exercises depending on the target force set for the individual.

The treatment was given for 6 sessions over two weeks, three times a week. No other treatment was given. Once the patient finished 6 sessions of eccentric training, outcome measures (i.e. percentage time on target, PSS and SF-36) were again recorded.

Statistical analysis was performed using SPSS 13. The statistical analysis included Student's paired t-test to find the difference of pre and post values for the percentage time on target and SF-36 health survey components. Wilcoxon signed ranks test was done to find the difference between pre and post pain values.

A probability level of $\mathrm{P}<0.05$ was considered to be significant.

\section{RESULTS}

Significant changes were seen in all the outcome measures.Percentage time on target showed an average increase of 23.6 (87\%) which was statistically significant $P<0.001$ (Table 1). In the SF-36 health survey physical component score showed an average increase of 10.9 (30\%), mental component score showed an average increase of 2.6 (6\%) and bodily pain component showed an average increase of 29.2 (64\%). All were statistically significant at $P<0.01$. (Table 2) Pain scores (PSS) showed an average increase of 16.39 (28\%) which was statistically significant (Table 3). Figure 1 represents the decrease in pain in the individual components of PSS which showed that there was a significant decrease in the pain following the exercise.

\section{DISCUSSION}

The objective of this study was to find the effect of eccentric isotonic strengthening of the quadriceps muscle in patients with patellofemoral pain.

Quadriceps strengthening plays an important role in the management of patellofemoral pain syndrome ${ }^{[24]}$. It can be done either by selective strengthening of the vastus medialis (VMO) or the general strengthening of quadriceps. Both approaches seem acceptable for rehabilitating patients with PFPS ${ }^{[20,25]}$. However it was seen that it may be appropriate to undertake exercises involving selective activation of the vastus medialis early in the rehabilitation process and there should not be over focus on selective activation before progressing

Table 2: Changes in the values of the components of SF-36 health survey

\begin{tabular}{ccccccc} 
Components of & \multicolumn{2}{c}{$\begin{array}{c}\text { Physical component score } \\
\text { SF-36 }\end{array}$} & \multicolumn{2}{c}{ Mental component score } & \multicolumn{2}{c|}{ Bodily pain component score } \\
Pre & Post & Pre & Post & Pre & Post \\
Mean (SD) & $36.69(4.34)$ & $47.64(3.37)$ & $41.63(6.50)$ & $44.28(3.12)$ & $45.40(13.23)$ & $74.60(11.26)$ \\
Mean difference & -10.94 & -2.64 & -29.20 \\
P value & $<0.001$ & 0.007 & $<0.001$
\end{tabular}

SD: Standard Deviation 


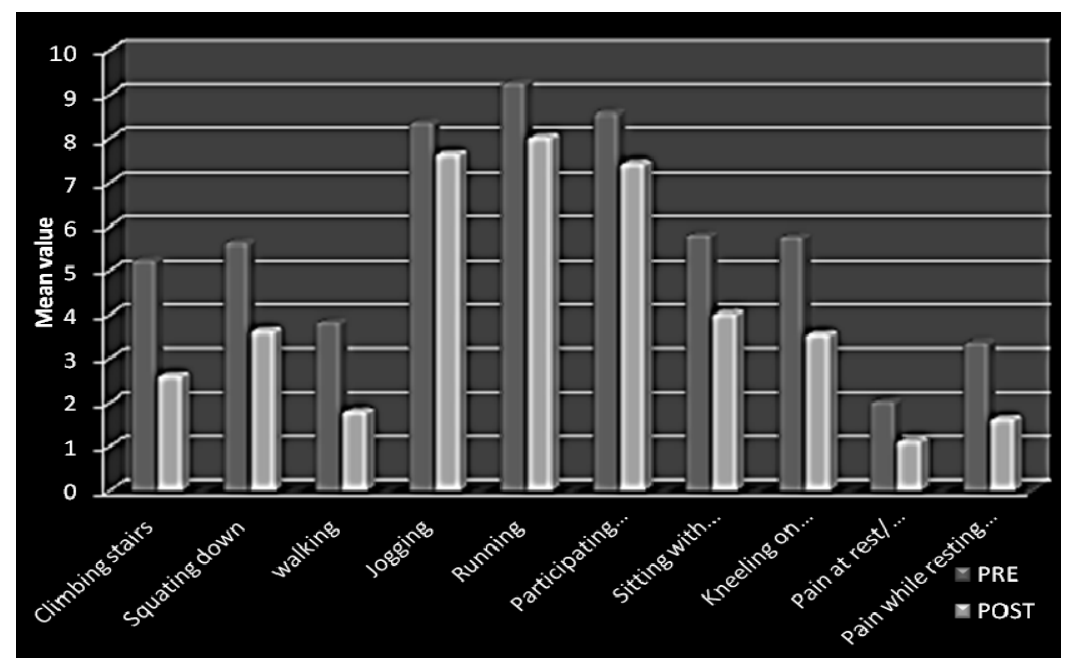

Fig. 1: Changes in the individual components of the patellofemoral pain severity scale

rehabilitation, especially in more chronic cases with significant participation restrictions ${ }^{[24]}$. Quadriceps retraining is, however, associated with good clinical outcomes in patients found to have VMO impairments [26]. Many authors have given much emphasis to selective recruitment of VMO for the management of the patellofemoral pain syndrome ${ }^{[2,27-29]}$ but some authors do conclude that VMO cannot be selectively recruited ${ }^{[30-32]}$. Evidence suggests that there exists considerable disagreement whether the VMO can be exercised in isolation ${ }^{[2]}$.

It has also been seen that generalized quadriceps exercises performed in the absence of other interventions (e.g. taping, bracing) may be effective in the management of patellofemoral pain and may be sufficient to relieve pain and reduce disability ${ }^{[33]}$. Therefore focusing on quadriceps would be a better approach rather than selective intervention. In our study we used the general strengthening of the quadriceps and found excellent results in all the outcomes measured.
It has been seen that each unit of work performed in the isotonic contractions of the quadriceps resulted in greater motor unit recruitment or an increased rate of firing, or both than in the isokinetic mode. This is important in the early phase of rehabilitation, when goals include complete motor unit recruitment of injured or atrophied muscles ${ }^{[34]}$. In our study though none of the patients were in the acute state we got good results in the performance of the quadriceps muscles as shown by the improvement in the percentage time on target. Hence isotonic exercises may be indicated in these patients as well.

Isotonic exercise has shown to be functional and play a major role in day to day activities. Though eccentric training has been given using exercises like progressive step down exercises and squats in one study and resulted in good outcome, it lacked objectivity to measure the improvement in eccentric control ${ }^{[17]}$. In our study eccentric training was given in the isotonic mode using BTe primus machine which allows the objective documentation of the

Table 3: Changes in the pain scores in Patellofemoral pain severity scale

\begin{tabular}{|cccc} 
& $\begin{array}{c}\text { Mean } \\
\text { (Standard Deviation) }\end{array}$ & $\begin{array}{c}\text { Mean } \\
\text { difference }\end{array}$ & P value \\
\hline Pre & $57.94(11.07)$ & 16.39 & 0.001 \\
Post & $41.55(15.08)$ & &
\end{tabular}


improvement by recording the increase in the percentage time on target. The percentage time on target which represents the percentage of the total treatment time that a subject will be able to control the target forces effectively also showed an increase in our study. This indicates that there was an increase in the eccentric strength of the muscles.

However when eccentric exercises are done muscles are less active as indicated by EMG records ${ }^{[7]}$. Thus the central nervous system can exploit the ability of muscles to generate higher tensions/myofiber during negative work and thereby reduce the energy cost by reducing the number of active motor units. Motor control thus plays an important role in the rehabilitation in general and patellofemoral joint problems in particular. Teaching the patient to better utilize the muscle stabilizers of the patella is the primary goal of many rehabilitative programs ${ }^{[7]}$. Previous studies have all demonstrated the body's ability to adapt rapidly and via motor learning to enhance both strength and function ${ }^{[35]}$. It demonstrated that when exercises were performed using biofeedback the recovery was faster compared to those without $\mathrm{it}^{[36]}$. In our study the feedback from the screen to control the movement and keep it within the target could have helped our patients. The current availability of advanced exercise equipment provides a promising environment for further investigation and clinical appliance.

Subjects showed an improvement in physical component score, mental component score and bodily pain as shown in SF-36 results which can be considered to be clinically significant. Probably better eccentric control of the quadriceps lead to the improvement in daily activities as reflected by improved physical component score. Pain showed a significant decrease in our group. Pain relief most probably could have been as a result of motor control which occurred during this time frame. Exactly how the training of the quadriceps relieved pain is unclear, this is in accordance with a similar type of study done before to improve the eccentric control of quadriceps muscle in patellofemoral pain syndrome ${ }^{[7]}$. One of the reasons that the pain might have been reduced is that the increase in the strength of the quadriceps muscle might have altered the contact location and pressure distribution, possibly relieving sensitive areas and thus relieving pain ${ }^{[37]}$. This also may be due to adaptive changes in the muscle because of eccentric training, which may reduce future tissue damage and pain ${ }^{[31]}$.

This study has shown that if patients with patellofemoral pain are trained in eccentric exercises of the quadriceps, pain decreases, the physical and mental components of the SF-36 form show an improvement and the percentage time that the patient is able to control the target also increases. Thus isotonic eccentric quadriceps exercises should form a part of treatment in the rehabilitation protocol for the patients with patellofemoral pain syndrome.

However a few limitations were noted. A control group was not included and so the effects cannot be related to the intervention given, however it was done as a pilot study to see the effect of eccentric muscle strengthening in PFPS. Further studies should be done with a control group. Though the percentage time on target showed significant results, the increase in the eccentric control of the quadriceps can be further documented by doing Functional Performance Tests like step down. Also the long term effect of the intervention was not assessed. The eccentric control of the hip musculature also seems to play role in the management of PFPS but we did not assess those muscles. Future studies could be done studying the isotonic eccentric control of the hip muscles on the PFPS.

\section{CONCLUSION}

Quadriceps femoris muscle strengthening is useful for improving functional ability, in patients with PFPS. Isotonic eccentric training of quadriceps muscles is effective in reducing pain and improving the functional status of patients with patellofemoral pain syndrome and can be suggested as part of treatment.

Conflict of interests: None 


\section{REFERENCES}

1. Wilk K, Davies G, Mangine R, Malone T. Patellofemoral disorders: A classification System and Clinical Guidelines for Nonoperative Rehabilitation. J Orthop Sports Phys Ther 1998;28:307-22.

2. Powers CM. Rehabilitation of patellofemoral joint disorders: a critical review. J Orthop Sports Phys Ther 1998;28:345-54.

3. Thein J, Brody LT. Nonoperative treatment for patella femoral pain. J Orthop Sports Phys Ther 1998;28:336-44.

4. Dixit S, Difiori J, Burton M, Mines B. Management of Patellofemoral Pain Syndrome. Am Fam Physician 2007;75:194-202.

5. Johnston L B, Gross M T. Effects of Foot Orthoses on Quality of Life for Individuals with Patellofemoral Pain Syndrome. J Orthop Sports Phys Ther 2004;34:440-48.

6. Powers CM. Patellar kinematics, part II: the influence of the depth of the trochlear groove in subjects with and without patellofemoral pain. Phys Ther 2000;80(10):965-78.

7. Bennett, Stauber J. Evaluation and treatment of anterior knee pain using eccentric exercise. Med Sci Sports Exerc 1986;18: 526-30.

8. Anderson G, Herrington L. A comparison of eccentric isokinetic torque production and velocity of knee flexion angle during step down in patellofemoral pain syndrome patients and unaffected subjects. Clin Biomech 2003;18:500-04.

9. Olmo J,. Aguado JP, Cortés L, et al. Quadriceps strength loss at flexed knee angles in active high-level soccer players with patellofemoral pain. Isokinet Exerc Sci 2007;15:303-07.

10. Callaghan MJ, Oldham JA. Quadriceps atrophy: to what extent does it exist in patellofemoral pain syndrome? Br J Sports Med 2004;38:295-99.

11. Werner S, Eriksson E. Isokinetic quadriceps training in patients with patellofemoral pain syndrome. Knee Surg Sports Traumatol Arthrosc 1993;1:162-68.

12. Alaca R, Yilmaz B, Goktepe AS, et al. Efficacy of isokinetic exercise on functional capacity and pain in patellofemoral pain syndrome. Am J Phys Med Rehabil 2002;81:807-13.

13. Thomeé R, Renström P, Karlsson J, Grimby G. Patellofemoral pain syndrome in young women. II. Muscle function in patients and healthy controls. Scand J Med Sci Sports 1995;5:245-51.

14. Adams W B.Treatment Options in Overuse Injuries of the Knee: Patellofemoral Syndrome, Iliotibial Band Syndrome, and Degenerative Meniscal Tears. Curr Sports Med Rep 2004;3:256-60.

15. Post W R. Patellofemoral pain results of nonoperative Treatment. Clin Orthop Relat Res 2005;436:55-59.

16. Powers CM, Pemy J, Hsu A, Hislop HJ. Are Patellofemoral Pain and Quadriceps Femoris Muscle Torque Associated With Locomotor Function? Phys Ther 1997;77:1063-69.

17. Clark DI, Downing N, Mitchell J, et al. Physiotherapy for anterior knee pain: a randomised controlled trial. Ann Rheum Dis 2000;59:700-04.

18. Mason M, Keays SL, Newcombe PA. The effect of taping, quadriceps strengthening and stretching prescribed separately or combined on patellofemoral pain. CPhysiother Res Int 2011;16:109-19.

19. Crossley K, Bennell K, Green S, et al. Physical therapy for patellofemoral pain: a randomized, double-blinded, placebo-controlled trial. Am J Sports Med 2002;30(6):857-65.

20. Syme G, Rowe P, Martin D, Daly G. Disability in patients with chronic patellofemoral pain syndrome: a randomised controlled trial of VMO selective training versus general quadriceps strengthening. Man Ther 2009;14:252-63.

21. Thomee R.A Comprehensive Treatment Approach for Patellofemoral Pain Syndrome in Young Women. Phys Ther 1997;77:1690-703.

22. Sanchis V Alfonso, Prat JM Pastor, et al. Biomechanical bases for Anterior Knee Pain and Patellar Instability in the young patient. In: Sanchis V Alfonso (ed). Anterior Knee Pain and patellar instability. $1^{\text {st }}$ ed. London: Springer Verlag. 2006; Pp:55-76.

23. Laprade JA, Culham EG. A self-administered pain severity scale for patellofemoral pain syndrome. Clin Rehabil 2002;16:780-8.

24. Witvrouw E, Cambier D, Danneels L, et al. The effect of exercise regimens on reflex response time of the vasti muscles in patients with anterior knee pain: a prospective randomized intervention study. Scand J Med Sci Sports 2003;13:251-8.

25. Bennell K, Duncan M, Cowan S, et al. Effects of Vastus Medialis Oblique Retraining versus General Quadriceps Strengthening on Vasti Onset. Med Sci Sports Exerc 2010;42:856-64.

26. Fagan V, Delahunt E. Patellofemoral pain syndrome: a review on the associated neuromuscular deficits and current treatment options. Br J Sports Med 2008;42:489-95.

27. Owings TM, Grabnie MD. Motor control of the vastus medialis oblique and vastus lateralis muscle is distrupted during eccentric contractions in subjects with patellofemoral pain. Am J Sports Med 2002;30:483-7.

28. Sinead p, Sullivan O. Activation of vastus medialis obliques in patients with patellofemoral pain syndrome. $J$ Strength Cond Res 2005;19:302-4.

29. Cerny K. Vastus Medialis Oblique/Vastus Lateralis Muscle Activity Ratios for Selected Exercises in Persons With and Without Patellofemoral Pain Syndrome. Phys Ther 1995;75:672-83. 
30. Malone T, Davies G, Walsh W. Muscular control of the patella. Clin Sports Med 2002;21:349-62.

31. Mei-Hwa Jan, Tung-Ching Wei, Chen-Yi Song. Comparisons of quadriceps strength training, taping, and stretching on clinical outcomes in patients with Patellofemoral Pain Syndrome. J Biomech. 2007:40(S2).

32. Waryasz GR, McDermott AY. Patellofemoral pain syndrome (PFPS): a systematic review of anatomy and potential risk factors. Dyn Med 2008;7:9.

33. Brukner P, Khan K, Crossley K, et al. Anterior Knee Pain. In: Brukner P, Khan K. (ed) .Clinical Sports Medicine 3rd ed. New Delhi: Tata McGraw-Hill. 2007; Pp:506-37.

34. Randy J. Schmitz; Kevin C. Westwood. Knee Extensor Electromyographic Activityto- Work Ratio is Greater With Isotonic Than Isokinetic Contractions. J Athl Train 2001;36:384-7.

35. Rice J, Bennett G, Ruhling R. comparision of two exercises on VMO and VL EMG activity and force production. Isokinet Exerc Sci 1995;5:61-7.

36. Yip SL, Ng GY. Biofeedback supplementation to physiotherapy exercise programme for rehabilitation of patellofemoral pain syndrome: a randomized controlled pilot study. Clin Rehabil 2006;20:1050-57.

37. Y Yildiz, T Aydin, U Sekir, et al. Relation between isokinetic muscle strength and functional capacity in recreational athletes with chondromalacia patellae. Br J Sports Med 2003;37:475-79. 